\title{
Pulse shape discrimination in nonaromatic plastics
}

\author{
H. Paul Martinez, Iwona Pawelczak, Andrew M. Glenn, M. Leslie Carman,
} Natalia Zaitseva, and Stephen Payne

5 Lawrence Livermore National Laboratory, 7000 East Ave. L-188, Livermore, CA 94550-9698

Abstract

Recently it has been demonstrated that plastic scintillators have the ability to distinguish neutrons from gamma rays by way of pulse shape discrimination (PSD).

10 This discovery has lead to new materials and new capabilities. Here we report our work with the effects of aromatic, non-armatic, and mixed aromatic/ non-aromatic matrices have on the performance of PSD plastic scintillators.

Keywords: Pulse shape discrimination, plastics, scintillators, aromatic, non-aromatic

\section{Introduction}

Pulse shape discrimination (PSD) is a technique traditionally used for detection and disambiguation of high-energy neutrons in the presence of a gamma radiation background. The method is based on the difference in the shapes of the scintillation

20 signals due to excitation by neutrons (recoil protons) and $\gamma$-rays (recoil electrons) in organic scintillators. According to a commonly accepted mechanism, both gammaand neutron-induced pulses contain a short (prompt) and a long (delayed) fluorescence decay components.[1, 2] The prompt component results from the direct emission from excited singlet states, while the triplet-triplet interaction,

25 which leads to an Auger upconversion process, mediates the slow component. This process produces the excitation of additional singlet states decaying within the same spectral region, but over a longer and delayed timescale. The different proportions of the prompt and delayed components in scintillation pulses produced by neutron and gamma interactions give the basis for the pulse separation used in 30 PSD techniques. 
Recently Zaitseva et. al. demonstrated that the PSD scintillation phenomenon is possible in plastic matrix [3]. Since then there has been much interest and research on plastic PSD scintillators [4-11]. However, the basic requirements are still not completely understood, although it was shown that there is an excited state 35 concentration dependence needed for triplet-triplet annihilation to occur. Plastic scintillators require the highest threshold concentration of dye, on the order of 20$30 \%$ of a primary scintillation dye in order to facilitate triplet migration. This may be compared to liquid scintillators, which only require $1-3 \%$ of a dye likely owing to the translation of the triplet molecules such that they are able to encounter each 40 other.

Similar discrimination properties were also found in organic liquid scintillators, which typically are binary or ternary systems composed of highly efficient fluorescent dyes (e.g. p-terphenyl, PPO, PBD, or POPOP) dissolved in aromatic

45 solvents (e.g. xylene or PXE) [1,12]. Matrix compositions of scintillating plastics are similar to those used for the liquid scintillators, with the difference being solvents are replaced by aromatic polymers, such as polystyrene (PS) or polyvinyltoluene (PVT). For traditional low dye concentration scintillators, an aromatic matrix is a necessity. However, we contend that the PSD process can function efficiently in high

50 dye loaded systems in the absence of an aromatic matrix.

For multiple component scintillators, the largest factor in attaining good performance is maximizing the energy transfer process from each component. The process can be simply described as: absorption of energy by the matrix from the electrons and holes created by the ionizing radiation, which is then transferred to

55 the primary dye, followed by the transfer to a wave-length-shifting secondary dye. Throughout this three-step process, there is always the possibility of direct excitation of either dye, thereby requiring no transfer. Direct excitation is always a less efficient process, but in systems contain a higher dye concentration the probability for triplet-triplet interaction necessary for PSD is increased.

60 It is expected that introducing a non-aromatic component can degrade the PSD performance of a plastic scintillator, but in the case of plastics it can also 
provide mechanical enhancements to the plastic. PS has an average softening point of $80^{\circ} \mathrm{C}$, a bulk $\mathrm{UV}$ absorption that begins at approximately $380 \mathrm{~nm}$, and it measures 68-70 for Rockwell $\mathrm{M}$ hardness. Polymethyl-methacrylate (PMMA) is a non65 aromatic, transparent polymer first developed in the 1930's under the name Plexiglas. PMMA has a higher softening point, $125^{\circ} \mathrm{C}[13]$ and its bulk absorption does not begin until approximately $300 \mathrm{~nm}$. PMMA is also a harder plastic measuring 85-105 on the Rockwell M hardness scale. All of these mechanical and optical properties make PMMA an attractive candidate as a non-aromatic co-polymer to

70 enhance the properties (mechanical and optical) of a PSD plastic scintillator. This is even more desirable when considering the scintillation dye load necessary for efficient PSD in a plastics scintillator. Initial results showed that $30 \%$ and greater dye loads in pure PVT or PS could lead to soft and slightly opaque plastics, which can fail over time. The use of PMMA can allow for stabilization of the high dye loads

75 required in order to maximize the PSD performance [8, 14].

Since there have been no reported PSD plastic or liquid scintillators prepared in non-aromatic matrices, herein we report our work on plastics PSD scintillators prepared in aromatic, combined aromatic-non-aromatic, and non-aromatic matrices.

\section{Experimental}

Chemicals: 2,5-diphenyloxazole(PPO) 99\%, divinylbenzene, 80\% technical grade (DVB), methyl methacrylate 99\% ,1,1-Bis(tert-butylperoxy)-3,3,585 trimethylcyclohexane $92 \% \quad$ (L-231), styrene 99.5\%, 1,4-Bis-(2methylstyryl)benzene (Bis-msb) were purchased from Sigma-Aldrich and Acros organics respectively. All chemicals were used without further purification. Liquids were degassed by sparging with argon and solids were degassed by vacuum. Casting: All castings for 1 -inch diameter were done using $20 \mathrm{~mL}$ scintillation vials.

90 General polymerization: All procedures with liquid or plastic preparations were conducted under oxygen-free conditions in an Ar-filled glove box or in tightly sealed vessels. 1-inch diameter samples were prepared as 10g polymerizations. $3 \mathrm{~g}(13.5$ 
mmol) 2,5-diphenyloxazole (PPO) and 20mg(0.06 mmol) 1,4-Bis-(2methylstyryl)benzene (Bis-msb) were place in a $20 \mathrm{ml}$ scintillation vial. The 95 contents were then degassed in the anti-chamber of the glove box. Inside the glove box the appropriate ratio of styrene and methyl methacrylate were then added. A vortex mixer and a small amount of heat (via a hotplate) were used to dissolve the solids. Once the solution appeared to be homogeneous, $30 \mathrm{mg}(0.09 \mathrm{mmol})$ of L-231 was added to the solutions and the vials were sealed using vinyl tape and placed in

100 an oven under $\mathrm{N}_{2}$ at $50^{\circ} \mathrm{C}$. The temperature was then raised to $75^{\circ} \mathrm{C}$ over the period of 6 days. Samples were then removed from the oven and left to cool to room temperature. The vials were then scored using a diamond scribe and the glass broken. Sample were removed, sized and polished using the appropriated abrasives.

105 PSD Scintillation: Neutron detection properties of samples were studied, as descried previously[3], using a ${ }^{252} \mathrm{Cf}$ source shielded with $5.1 \mathrm{~cm}$ of lead, to irradiate samples coupled to Hamamatsu R6231-100-SEL photomultiplier tube (PMT). Gamma-equivalent energy calibration was made based on the position of the Compton edge in the ${ }^{137} \mathrm{Cs}$ spectra, in which 500 keVee was defined by $50 \%$ of the Compton edge peak. Relative

110 light yield (LY) of samples was evaluated from the position of the ${ }^{137} \mathrm{Cs}$ Compton edge and measured in arbitrary units, corresponding to channels. The signals collected at the PMT anode were recorded using a 14-bit high-resolution waveform CompuScope 14200 digitizer with a sampling rate of $200 \mathrm{MS} / \mathrm{s}$, for offline analysis. The waveforms were numerically integrated over two time intervals: $\Delta \mathrm{t}_{\text {Total }}$ and a subinterval $\Delta \mathrm{t}_{\text {Tail }}$, 115 corresponding to the total charge $\left(\mathrm{Q}_{\text {Total }}\right.$ and the delayed component $\left(\mathrm{Q}_{\text {Tail }}\right)$ of the signal, respectively. The value of the ratio of charge $\mathrm{R}=\mathrm{Q}_{\text {Tail }} / \mathrm{Q}_{\text {Total }}$ for the two time intervals indicated whether the considered event was likely produced by a neutron (high $\mathrm{R}$ value) or a gamma ray (small $\mathrm{R}$ value). Quantitative evaluation of PSD was made using Figures of Merit $F O M=S /\left(\delta_{1}+\delta_{2}\right)$, where $S$ is the separation between the peaks corresponding to

120 different types of radiation, and $\delta_{1}$ and $\delta_{2}$ are full width at half maxima (FWHM) of the corresponding peaks. 


\section{Results and Discussion}

125 As shown by Zaitseva et al. in plastic matrices the concentration threshold for efficient PSD is roughly 10 times of that in a liquid scintillator. This is due to the molecules moving in liquid and therefore come in contact with each other, while the triplets have to migrate from molecule to molecule for the plastics, thus necessitating the demand for higher concentrations. With this requirement there

130 are very few scintillating dyes that are soluble at the necessary concentration. Furthermore, the emission of the primary dye must be red-shifted from the matrix bandedge, and function well with a secondary, wave-shifting dye. Given these concentration and solubility requirements, PPO is the most efficient dye so far found for high concentration use in aromatic matrices. The traditional polymers used are

135 poly-vinyltoluene (PVT) and poly-styrene (PS), both are well suited for use as a scintillation matrix. We have chosen to use PS, because the resulting scintillators have shown improved performance, compared to PVT. The non-aromatic polymer chosen was poly-methyl methacrylate. PPO is equally soluble in methyl methacrylate as in styrene, is UV transparent in the desired regions, and yields a

140 transparent plastic. As previously stated, PMMA is a harder plastic than PS or PVT. This can be beneficial for machining and shaping plastics to meet specific needs. While these characteristics of PMMA can lead to a plastic with superior mechanical properties, it was unclear how significant the scintillation performance would deteriorate due to the lack of aromatics in the matrices.

145 In order to better understand how the incorporation of a non-aromatic matrices would affect the scintillation properties a series of plastics were prepared using a dye load of 30\%PPO and $0.2 \%$ bis-msb, and the ratio of PS to PMMA was increase in $10 \%$ increments. In addition to monomers, $5 \%$ (wt/wt) of divinylbenzene (DVB) was added to the co-polymers as a crosslinking agent. The

150 crosslinking of the plastic will lead to harder material, and can also stabilize the plastic by creating a tighter polymeric network. The sample preparation of $100 \%$ PMMA did not contain DVB, so that the matrices would be completely non-aromatic. In order to compare the samples as equivalently as possible, all samples were sized 
to $25 \mathrm{~mm}$ (od) $\times 14 \mathrm{~mm}(\mathrm{l})$. As expected the samples containing larger ratios of 155 PMMA: PS had greater densities, ranging from $1.19-1.02 / \mathrm{cm}^{3}$ (as seen in table 1).

Table 1. Density and light yield for mixed matrices PSD plastics composed of $30 \%$ PPO and 0.2\% Bis-msb

\begin{tabular}{|l|l|c|}
\hline Matrix & $\begin{array}{l}\text { Density } \\
(\mathrm{g} / \mathrm{cc})\end{array}$ & Light Yield (a.u) \\
\hline PMMA & 1.193 & 32 \\
\hline 9PMMA:1PS & 1.080 & 33. \\
\hline 8PMMA:2PS & 1.066 & 34 \\
\hline 7PMMA:3PS & 1.069 & 35 \\
\hline 6PMMA:4PS & 1.061 & 35 \\
\hline 5PMMA:5PS & 1.044 & 36.6 \\
\hline 4PMMA:6PS & 1.057 & 38 \\
\hline 3PMMA:7PS & 1.050 & 38.4 \\
\hline 2PMMA:8PS & 1.029 & 38.4 \\
\hline 1PMMA:9PS & 1.017 & 39.5 \\
\hline PS & 1.021 & 40 \\
\hline
\end{tabular}

160
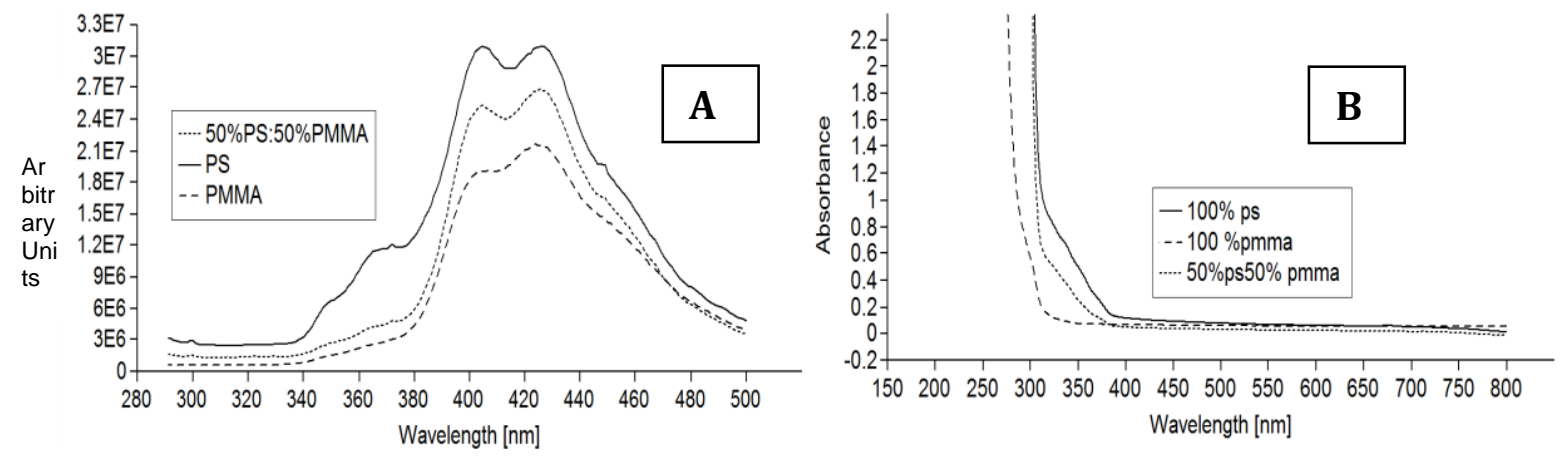

Figure 1. A shows the fluorescence emission of the plastics composed of $30 \%$ PPO and $0.2 \%$ bis-msb in PS, PMMA and mixed matrices and $\mathbf{B}$ shows UV/ Vis absorption edge of corresponding bulk plastic matrices without dyes.

165

The fluorescence spectra were taken using $275 \mathrm{~nm}$ as the excitation wavelength for the sample in PS and an excitation wavelength of $300 \mathrm{~nm}$ for the samples in PMMA and 1:1 mixture of PS: PMMA (as seen in fig. 1A). The emission was measured for the range of $300-500 \mathrm{~nm}$. The reason for the two different excitation wavelengths is

170 that the sample in PMMA cannot transfer the energy to the dye when the matrix is excited. This is why the PS has a small shoulder at approximately $360 \mathrm{~nm}$ 
corresponding to the emission of PPO. The PPO emission is not seen in PMMA, since it is able to transfer its energy to the secondary dye. As seen in fig. 2B the absorption edge of the pure plastic shifts to lower wavelengths as the PMMA concentration is

175 increased. At 50\%PMMA bulk absorption edge has been shifted by approximately $20 \mathrm{~nm}$ and when PS is completely absent the absorption edge is approximately at $300 \mathrm{~nm}$.

The light yield of our samples are comparable to the commercially available, EJ-299-33 PSD plastic. As seen in table 1, a light yield of 32 a.u was recorded for the 180 sample in pure PMMA and 40a.u for the sample in pure PS. The light yields of the samples composed of mixtures of PS -PMMA's increase as the concentration of PS increase, as expected (as seen in fig. 2A). The same is seen of the figure of merit (FoM). What was somewhat unexpected was the difference in the two extreme cases. Only 20\% difference in light yield between the sample comprised of a PS

185 (aromatic) and PMMA (non-aromatic) matrices was recorded. This is likely due to the high concentration of PPO and could suggest that in systems of high dye load the matrices role may be lessened. In the absence of an aromatic matrix,

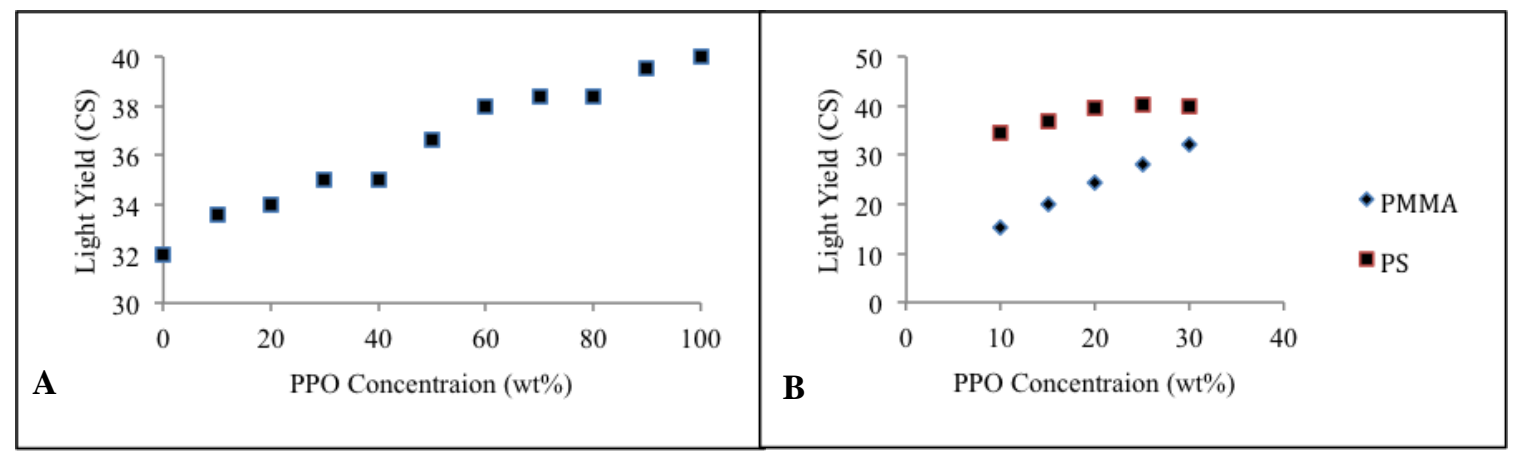

Figure 2 A shows the relationship between the polystyrene concentration and the light yield (Cs). B shows the light yield (Cs) as a function of the PPO concentration 190 for pure PMMA and pure PS.

PPO may form its own network, which can function in a similar fashion. This is more easily seen in samples where the concentration is gradually increased (as seen in fig. 2B). In samples in PS the effects of the PPO concentration are small; the total range of the light yield is 34.4-40 in PS and for PMMA 15.2-32 (arbitrary units). This is $19514 \%$ vs. $52.5 \%$ change in scintillation light yield for PS and PMMA (respectively). 
There is no direct connection of how the scintillation light yield affects the PSD performance, although having high light yield in PSD material is always desirable. The PSD, first and foremost, is superior in plastics samples prepared in PS or PVT, but the performance does not dramatically degrade when PS is replaced with PMMA. The difference in the PSD figure of merit (FoM) in samples in PMMA vs PS is $24 \%$.
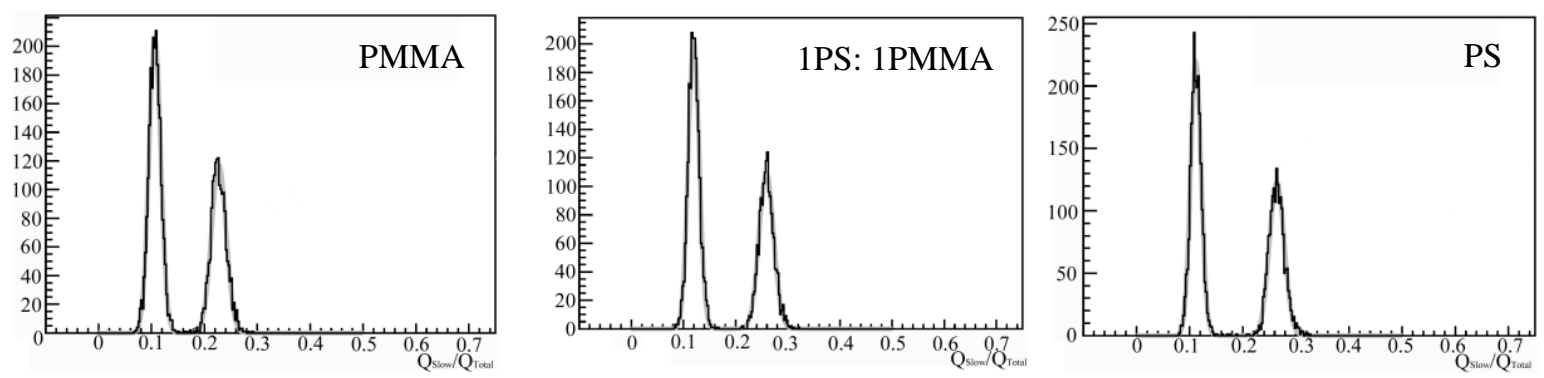

Figure 4 shows 2D-PSD plots of plastic samples prepared in PS, PMMA and a 1:1 mixture of PS and PMMA in the $\gamma$-equivalent energy 412-582keVee.

As seen above in fig. 4, the replacement of PS with PMMA degrades the PSD, but deterioration is gradual. 1:1 ratio of PMMA to PS compared to pure PS has difference in FoM is $12 \%$ and a sample's FoM prepared in PMMA is $12 \%$ worse. This is an overall of $24 \%$ loss when utilizing a completely non-aromatic matrix. A more

210 complete trend can be seen in fig. 5, where the PS concentration was replaced in $25 \%$ increments to better see how the loss of an aromatic matrix affected the PSD. Similar effects were seen when analogs were prepared using $p$-xylene for PS and ethyl acetate (EtOAc) for PMMA. The trend in the liquids was very much similar to that of what was shown in the plastic system. The separation between neutrons and 215 gammas deteriorates with the loss of the aromatic matrices, but in non-aromatic matrices there is still clear separation (can be seen in supplemental data). 


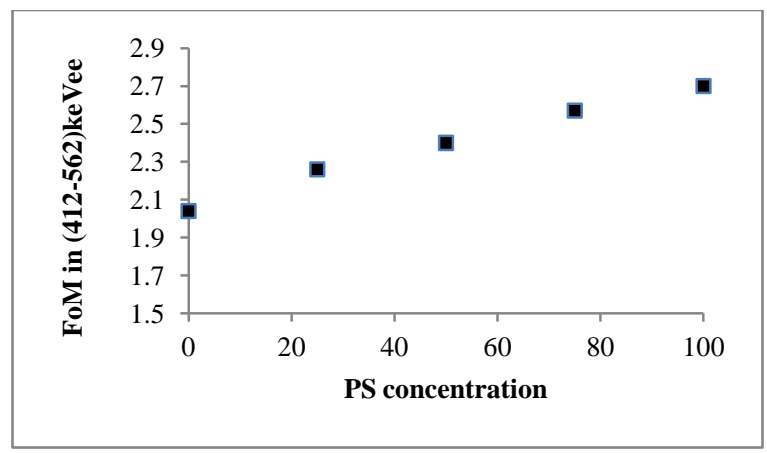

220 Figure 5 shows the PSD FoM of five plastic samples in which the concentration is changed in $25 \%$ increments. The $0 \%$ corresponds to a PMMA matrix.

In order to confirm that this phenomenon was not unique to PPO, samples were prepared using $m$-terphenyl as a primary dye and several secondary dyes in PMMA.

$225 m$-Terphenyl is not a traditional scintillation dye due to its low quantum yield (29\%) and its absorption lies below that of PS, rendering it unusable in aromatic plastics. It does scintillate and is soluble at the high levels needed to function in a plastic scintillator. We found that at high concentrations these samples too were capable of PSD (as reported in the supplemental data) and its scintillation light output can be 230 enhanced with a secondary dye similar to an aromatic system.

\section{Conclusion}

We have found that plastic PSD scintillators synthesized in non-aromatic and aromatic/non-aromatic mixtures can perform reasonably compared to their 235 aromatic counter parts. While the PSD is superior in completely aromatic matrices the use of a co-polymeric systems can be beneficial. Non-aromatic polymers can allow for the use of dyes previously incompatible. Whether the problem is insolubility or matrix absorption, PMMA allows for investigation of new compounds. Furthermore, when the mass fraction of the matrices is aromatic, small amounts of 240 PMMA can provide important mechanical enhancement, while having minimal effect on the overall performance of the scintillator. 
Acknowledgements. This work was supported by the U.S. DOE, NNSA, Office of Defense Nuclear Nonproliferation, Office of Nonproliferation Research and 245 Development (NA-22) and performed under the auspices of the U.S. Department of Energy by Lawrence Livermore National Laboratory under Contract DE-AC5207NA27344. LLNL-JRNL-654062

[1] J.B. Birks, The theory and practice of scintillation counting, (1964).

[2] F.D. Brooks, Development of organic scintillators, Nuclear Instruments and Methods, 162 (1979) 477-505.

255 [3] N. Zaitseva, B.L. Rupert, I. Pawelczak, A. Glenn, H.P. Martinez, L. Carman, M.

Faust, N. Cherepy, S. Payne, Plastic scintillators with efficient neutron/gamma pulse shape discrimination, Nuclear Instruments and Methods in Physics Research Section A: Accelerators, Spectrometers, Detectors and Associated Equipment, 668 (2012) 88-93.

260 [4] A. Favalli, M.L. Iliev, K. Chung, C. Hurlbut, H.P. Martinez, M.T. Swinhoe, N.P. Zaitseva, K.D. Ianakiev, Pulse Shape Discrimination Properties of Neutron-Sensitive Organic Scintillators, Nuclear Science, IEEE Transactions on, 60 (2013) 1053-1056. [5] P.L. Feng, J. Villone, K. Hattar, S. Mrowka, B.M. Wong, M.D. Allendorf, F.P. Doty, Spectral- and Pulse-Shape Discrimination in Triplet-Harvesting Plastic Scintillators, 265 Nuclear Science, IEEE Transactions on, 59 (2012) 3312-3319. [6] S. Nyibule, E. Henry, W.U. Schroder, J. Toke, L. Acosta, L. Auditore, G. Cardella, E. De Filippo, L. Francalanza, S. Giani, T. Minniti, E. Morgana, E.V. Pagano, S. Pirrone, G. Politi, L. Quattrocchi, F. Rizzo, P. Russotto, A. Trifiro, M. Trimarchi, Radioluminescent characteristics of the EJ 299-33 plastic scintillator, Nuclear Instruments and

270 Methods in Physics Research Section A: Accelerators, Spectrometers, Detectors and Associated Equipment, 728 (2013) 36-39.

[7] S.A. Pozzi, M.M. Bourne, S.D. Clarke, Pulse shape discrimination in the plastic scintillator EJ-299-33, Nuclear Instruments and Methods in Physics Research 
Section A: Accelerators, Spectrometers, Detectors and Associated Equipment, 723 275 (2013) 19-23.

[8] N. Zaitseva, A. Glenn, H. Paul Martinez, L. Carman, I. Pawelczak, M. Faust, S. Payne, Pulse shape discrimination with lithium-containing organic scintillators, Nuclear Instruments and Methods in Physics Research Section A: Accelerators, Spectrometers, Detectors and Associated Equipment, 729 (2013) 747-754.

280 [9] N.P. Hawkes, G.C. Taylor, Analysis of the pulse shape mechanism in a plastic scintillator with efficient neutron/gamma pulse shape discrimination, Nuclear Instruments and Methods in Physics Research Section A: Accelerators, Spectrometers, Detectors and Associated Equipment, 729 (2013) 522-526. [10] A.N. Mabe, J.D. Auxier Ii, M.J. Urffer, D. Penumadu, G.K. Schweitzer, L.F. Miller, 285 Transparent lithiated polymer films for thermal neutron detection, Nuclear Instruments and Methods in Physics Research Section A: Accelerators, Spectrometers, Detectors and Associated Equipment, 722 (2013) 29-33.

[11] A.N. Mabe, J.D. Auxier, M.J. Urffer, S.A. Young, D. Penumadu, G.K. Schweitzer, L.F. Miller, Thin Film Polymer Composite Scintillators for Thermal Neutron Detection, 290 Journal of Composites, 2013 (2013) 8.

[12] Ross, S. Organic, Liquid scintillation counting and organic scintillators, Lewis, Chelsea, Mich (1991).

[13] M. Kutz, Handbook of materials selection, J. Wiley, New York, 2002.

[14] I.A. Pawelczak, A.M. Glenn, H.P. Martinez, M.L. Carman, N.P. Zaitseva, S.A. Payne,

295 Boron-loaded plastic scintillator with neutron- $\gamma$ pulse shape discrimination capability, Nuclear Instruments and Methods in Physics Research Section A: Accelerators, Spectrometers, Detectors and Associated Equipment, 751 (2014) 6269. 